\title{
Design of Optimal Laser Fields to Control Vibrational Excitations in Carboxy-myoglobin
}

\author{
Harjinder Singh ${ }^{1}$, Sitansh Sharma ${ }^{1}$, Praveen Kumar ${ }^{2}$, Jeremy N. Harvey ${ }^{3}$, \\ and Gabriel G. Balint-Kurti ${ }^{3}$ \\ ${ }^{1}$ Center for Computational Natural Sciences and Bioinformatics, \\ International institute of Information Technology, Hyderabad 500032, India \\ laltu@iit.ac.in \\ ${ }^{2}$ Department of Chemistry, Panjab University, Chandigarh - 160014, India \\ ${ }^{3}$ Centre for Computational chemistry and School of Chemistry, \\ University of Bristol, Bristol BS8 1TS, UK
}

\begin{abstract}
Optimal control theory is applied to obtain infrared laser pulses for the selective vibrational excitation of a two mathematical dimensional model of carboxy-myoglobin. Density functional theory is used to obtain the potential energy and dipole moment surfaces of the active site model. The Conjugate gradient method is employed to optimize the cost functional and to obtain the optimized laser pulses. Optimized laser fields are found which give virtually $100 \%$ excitation probability to preselected vibrational levels.
\end{abstract}

\section{Introduction}

Application of control theory $123456 / 7891011$ to biological systems is quite difficult because of their large size and complexity. The availability of ultrafast and intense mid-IR pulses has opened the way to achieving laser control in molecules such as proteins. Hemoglobin and myoglobin are among the most studied proteins, especially the structure and dynamics of the Fe-CO bond have been extensively studied for several years 12 13 14 15. The possibility of laser control of dynamics in biological systems using frequencies in the visible regime has been established by various groups [15. Recently Joffre et al. have demonstrated coherent vibrational climbing up to $v=6$ state of $\mathrm{C}-\mathrm{O}$ vibration in carboxy-hemoglobin using mid-IR chirped ultrashort pulses [16].

Our aim is to use optimal control theory (OCT) to design laser pulses for the selective vibrational excitation of the Fe-C-O fragment of the active site of carboxy-myoglobin. Peirce et al. 11 introduced the concept of OCT in molecular control, in which the problem is posed as an optimization of a cost functional. We have applied optimal control theory for selective vibrational excitation of $\mathrm{Fe}-\mathrm{C}$ bond at the active site of carboxy-myoglobin. We have treated the interaction of the molecule with the electric field within the dipole approximation. As the dipole moment of the $\mathrm{C}-\mathrm{O}$ bond in the Fe-C-O fragment is much higher than that of the Fe-C bond, so the C-O dipole will interact more strongly with the applied laser field and hence will absorb more energy. A reasonable physical mechanism for the

M. Bubak et al. (Eds.): ICCS 2008, Part II, LNCS 5102, pp. 387-395. 2008.

(C) Springer-Verlag Berlin Heidelberg 2008 
excitation of the Fe-C vibrational motion would be to first deposit energy in the $\mathrm{C}-\mathrm{O}$ bond and then to selectively transfer this energy into the Fe- $\mathrm{C}$ bond. For the theoretical design of a laser field, we have performed optimal control calculations on Fe-C-O portion of the optimized structure. The potential energy and dipole moment surfaces are obtained using DFT calculations of a 60 atom model of the active site of the system. The Fe-C and $\mathrm{C}-\mathrm{O}$ separations are systematically varied while all other internal coordinates are held fixed at their values in the equilibrium geometry. The paper is organized as follows: In Sec. 2.1 we present the details of electronic structure calculations and the description of the active site in consideration. In Sec. 2.2 the optimal control formulation for triatomic system (Fe-C-O) is described in detail. Sec. 2.3 describes the conjugate gradient method, used for optimisation of the functional constructed in Sec. 2.2. In Sec. 3 , the results are discussed in detail and finally in Sec. 4 we conclude with a summary.

\section{Theory and Method}

\subsection{Electronic Structure Calculations and Active Site Model}

In the present study, ab initio electronic structure calculations are performed using the Gaussian03 suite [17] of quantum chemical programmes. In order to model the active site of carboxy-myoglobin, we chose hexa-coordinated ironporphyrin-imidazole-CO as our model structure, where the imidazole ligand is used to mimic the effect of the proximal histidine residue. Geometry optimization of this model structure was performed using density-functional theory (DFT), with the popular B3LYP hybrid extended-correlation functional. For iron, oxygen, nitrogen and carbon we have used the $6-311 \mathrm{G}^{*}$ basis set while the $6-31 \mathrm{G}$ basis was used for the hydrogen atoms. We have chosen Fe-C-O fragment to be our control system, treating the rest of the molecule as frozen. The crystallographic studies of carboxy-myoglobin (MbCO) show an almost linear FeCO geometry as well as a nearly perfect square planar symmetry with respect to the nitrogen plane formed by the four pyrrole N-ligands of the heme iron [18]. Figure 1 shows that the Fe-C-O fragment in the optimized structure is almost straight, so we have chosen the Fe-C-O fragment to be linear throughout our optimal field calculations. This is substantiated by the geometrical parameters obtained from the electronic structure calculations. The variations of potential energy and dipole moment with change in bond lengths (Fe-C, C-O) are shown in Fig. 2.

\subsection{Dynamical Evolution and Application of Optimal Control Theory}

In our control system (Fe-C-O) there are two bonds and the problem is formulated using Jacobi coordinates $\mathrm{R}$ and $\mathrm{r}$, shown in Fig. 1(b), where the coordinate $R$ is the distance of iron atom from center of mass of C-O bond and $r$ is the carbon oxygen bond length. Our aim is to design a laser pulse that can selectively 

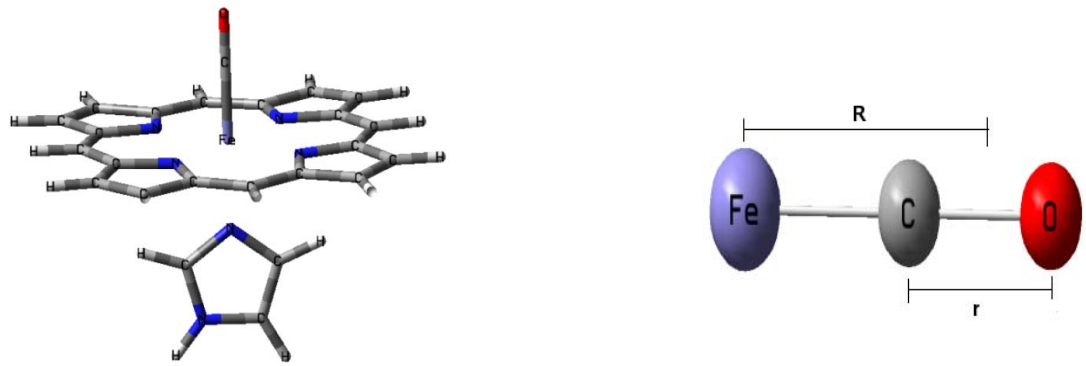

Fig. 1. (a) Geometry optimized hexa-coordinated active site model, obtained using DFT, where the imidazole ring mimics the effect of the proximal histidine residue; (b) Jacobi coordinate representation of linear triatomic fragment $\mathrm{Fe}-\mathrm{C}-\mathrm{O}$, where $\mathrm{R}=$ distance of $\mathrm{Fe}$ from center of mass of $\mathrm{C}-\mathrm{O}$ bond while $\mathrm{r}=$ bond length of $\mathrm{C}-\mathrm{O}$ bond

transfer population from an initial vibrational state to a desired target vibrational state at a fixed time. For the design of such a laser pulse we construct a cost functional containing our objective, a penalty term for the fluence and the dynamical constraint requiring that the time-dependent Schrödinger equation be obeyed at all times. The Grand cost functional to be optimized takes the following form:711

$$
J[\epsilon(t)]=\left|\left\langle\psi_{i}(T) \mid \phi_{f}\right\rangle\right|^{2}-\alpha_{0} \int_{0}^{T}[\epsilon(t)]^{2} d t-2 \operatorname{Re}\left[\int_{0}^{T}\left\langle\chi_{f}(t)\left|\frac{\partial}{\partial t}+i \hat{H}(\epsilon(t))\right| \psi_{i}(t)\right\rangle d t\right],
$$

where, the first term contains the physical objective, the second accounts for the penalties on pulse energy and the last term comes from the dynamical constraint. The function $\psi_{i}(t)$ is the initial wavefunction propagated to time $t$ under the action of the optimal laser field $\epsilon(t)$ and $\phi_{f}$ is the target state to be reached at the final time $T$. The function $\chi_{f}(t)$ can be regarded as a Lagrange multiplier introduced to assure satisfaction of the time-dependent Schrödinger equation. The factor $\alpha_{0}$ is a constant positive weighting parameter that specifies the weight of the pulse energy in the functional. The time evolution of the quantum state $|\psi(t)\rangle$ under the influence of an external field, $\epsilon(t)$, within the dipole approximation, is governed by the time-dependent Schrödinger equation (atomic units used throughout the paper, $\hbar=1$ ),

$$
i \frac{\partial}{\partial t}|\psi(t)\rangle=\hat{H}|\psi(t)\rangle=\left[\hat{H}_{0}-\hat{\mu} \epsilon(t)\right]|\psi(t)\rangle,
$$

where $\hat{H}$ and $\hat{\mu}$ are the system Hamiltonian and the dipole moment operator, respectively (see [7] for further details).

Variation of the grand functional (Eq. (1)) with small independent changes in the wave function, $\psi_{i}(t)$, the Lagrange multiplier, $\chi_{f}(t)$ and the electric field, $\epsilon(t)$, leads to the following set of nonlinear pulse design equations for vibrational control in Fe-C-O part of carboxy-myoglobin: 

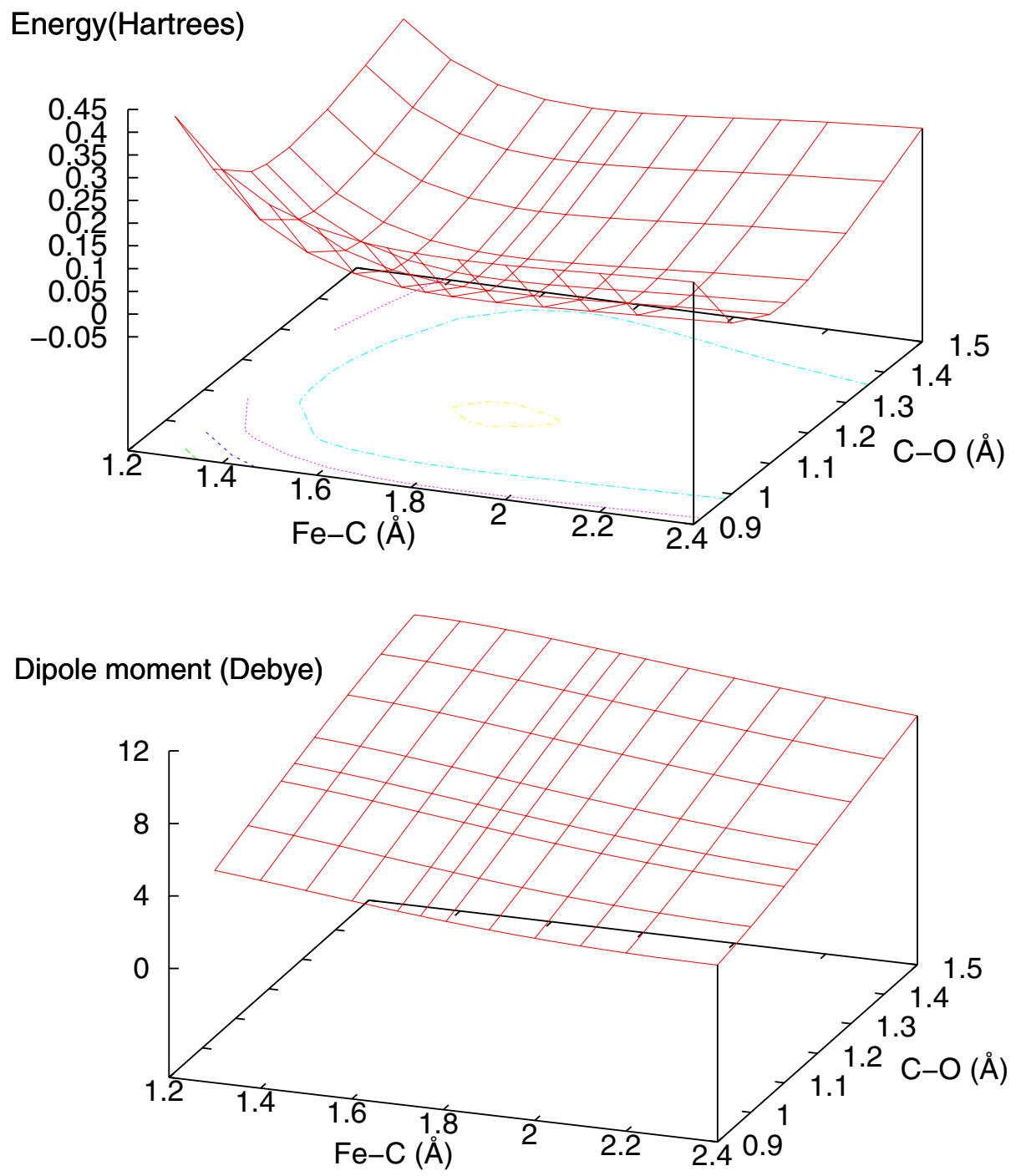

Fig. 2. Variation in potential energy and z-component (along Fe-C-O) of dipole moment with change in bond lengths of Fe-C and C-O bonds, obtained using DFT

$$
\begin{gathered}
i \frac{\partial \psi_{i}(t)}{\partial t}=\hat{H} \psi_{i}(t), \quad \psi_{i}(0)=\phi_{i}, \\
i \frac{\partial \chi_{f}(t)}{\partial t}=\hat{H} \chi_{f}(t), \quad \chi_{f}(T)=\left\langle\phi_{f} \mid \psi_{i}(T)\right\rangle \phi_{f}, \\
\alpha_{0} \epsilon(t)=\operatorname{Im}\left(\left\langle\chi_{f}(t)\left|\frac{\partial}{\partial \epsilon(t)} \hat{H}\right| \psi_{i}(t)\right\rangle\right) .
\end{gathered}
$$


These coupled differential equations can be solved iteratively. In order to maximize our cost functional (Eq. (1)),subject to Eqs. (3) and (4) we have used the conjugate gradient method discussed in detail in Sec. 2.3.

\subsection{Conjugate Gradient Method}

In order to theoretically design a laser pulse that can selectively control vibrational excitation in the iron-carbon part of carboy-myoglobin, calculations are carried out to maximize the value of the cost functional, $J$, mentioned in Eq. (1) using the conjugate gradient method [7. After $k$ number of iterations in the optimization cycle, the gradient of $J$ with respect to $\epsilon$ at time $t$ is given by

$$
\frac{\partial J^{k}}{\partial \epsilon^{k}(t)}=-2\left[\alpha_{0} \epsilon^{k}(t)-\operatorname{Im}\left\langle\chi_{f}(t)\left|\frac{\partial \hat{H}}{\partial \epsilon^{k}(t)}\right| \psi_{i}(t)\right\rangle\right] .
$$

In addition, a Gaussian envelope function $s(t)=e^{\frac{-(t-T / 2)^{2}}{(T / 4)^{2}}}$ is preserved throughout the optimization (see [7]) to ensure that the optimized field switches smoothly on and off. The wave function, $\psi_{i}(t)$, and the Lagrange multiplier, $\chi_{f}(t)$, are propagated in time using the second order split-operator method [19]. In order to determine the maximum value of the cost functional a line search is performed along the Polak-Ribiere-Polyak search direction calculated using Eq. (6) as

$$
d^{k}\left(t_{i}\right)=g^{k}\left(t_{i}\right)+\zeta^{k} d^{k-1}\left(t_{i}\right)
$$

where

$$
\zeta^{k}=\frac{\sum_{i} g^{k}\left(t_{i}\right)^{T}\left(g^{k}\left(t_{i}\right)-g^{k-1}\left(t_{i}\right)\right)}{\sum_{i} g^{k-1}\left(t_{i}\right)^{T} g^{k-1}\left(t_{i}\right)} ;
$$

$k=2,3, \ldots$, and $d^{1}\left(t_{i}\right)=g^{1}\left(t_{i}\right)$. To prevent the algorithm from sampling $\epsilon(t)$ values outside of the desired field amplitude range $\left[\epsilon_{\text {min }}, \epsilon\right]$ during the line search, the direction $d^{k}(t)$ is projected. The projected search direction is Fourier transformed to obtain a function of frequency, and this function is filtered using a $20^{t h}$-order Butterworth bandpass filter in order to restrict the frequency components of the electric field within a specified range. The field is updated using the following expression as

$$
\epsilon^{k+1}(t)=\epsilon^{k}(t)+\lambda d_{p}^{k}(t),
$$

where $\lambda$ is determined by the line search.

\section{Results and Discussion}

We discuss now the results obtained using the optimal control formalism described above, for two vibrational excitations of the molecule. The first excitation is from the ground vibrational state to the vibrational state corresponding to a C$\mathrm{O}$ stretch $(v=0 \rightarrow v=5)$. The second is an excitation from ground vibrational 

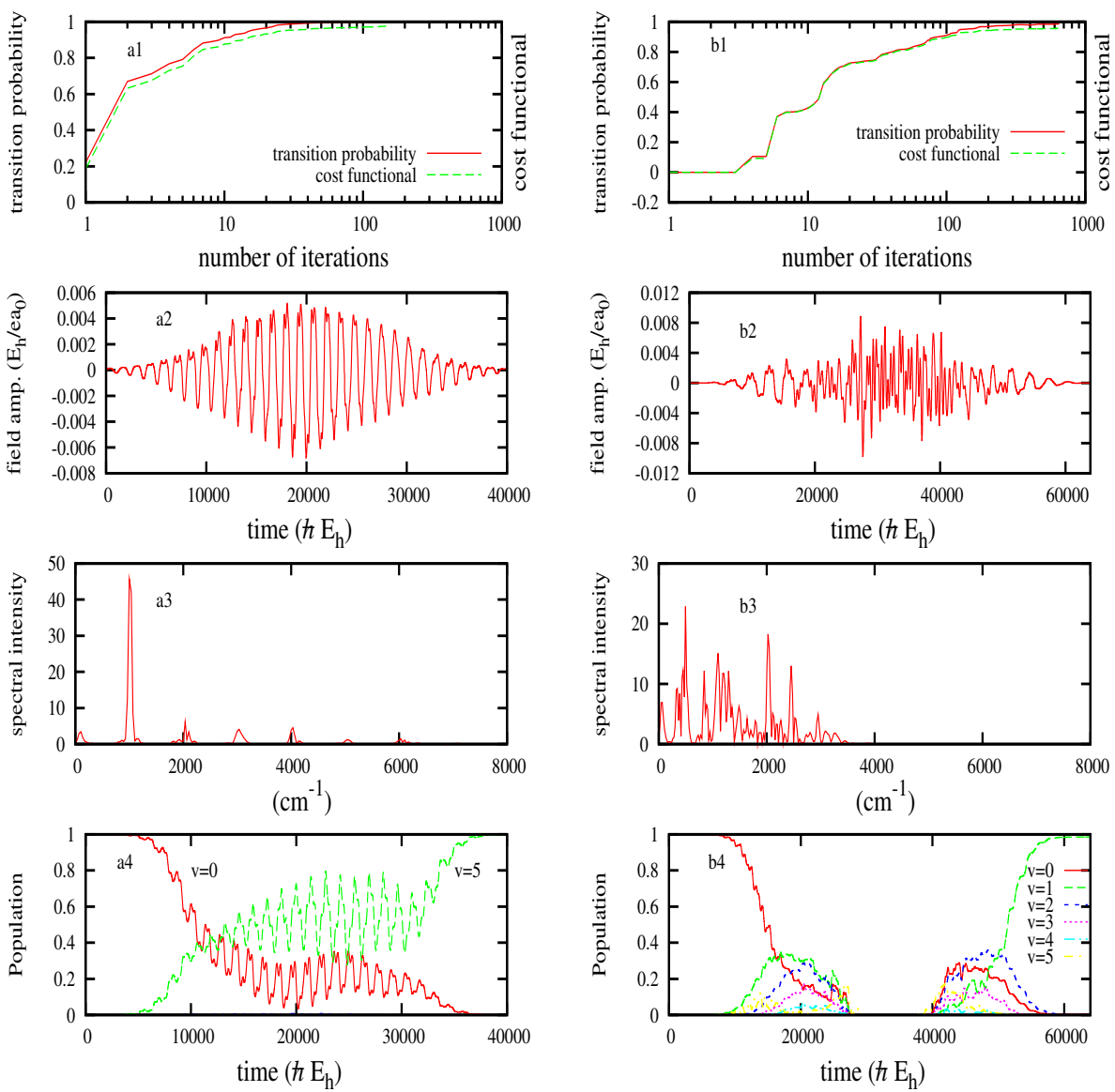

Fig. 3. Results for transitions $v=0$ to $v=5$ and $v=0$ to $v=1$ : (a1, b1) Convergence behavior of transition probability and the cost functional, J, with number of iterative steps involved in optimization; (a2, b2) optimized laser field and (a3, b3) its frequency distribution and $(\mathrm{a} 4, \mathrm{~b} 4)$ populations of different states as a function of time

state to the vibrational state corresponding to the Fe-C stretch $(v=0 \rightarrow v=1)$. The Fourier Grid Hamiltonian method [20] is used to create a direct product basis for the variational calculation of the triatomic vibrational wave functions of the (Fe-C-O) system. For the time propagation, we employed 64 grid points for both $\mathrm{R}$ and $\mathrm{r}$ coordinates. In order to impose constraints on the total laser fluence a constant penalty parameter $\alpha_{0}$ in Eq. (1) is set to 0.1 for both the transitions.

(a) Transition: $v=0 \rightarrow v=5$ : For this transition we have propagated the system (Fe-C-O) using a pulse of length $40,000 \hbar / E_{h}$, which is divided into 16384 time steps. The initial guess laser field is chosen as $\epsilon(t)=0.0075 \cdot \sin \left(\frac{\omega_{0,5}}{2} t\right) s(t)$ where $\omega_{0,5}$ is transition frequency corresponding to the $v=0 \rightarrow v=5$ vibration 
and $\mathrm{s}(\mathrm{t})$ is the envelope function (see above). We illustrate the results obtained in Fig. 3(a1-a4). The convergence of the transition probability and cost functional with the number of iterations steps involved in the optimization is shown in plot a1. The figure shows that the dynamical goal is reached perfectly with near $100 \%$ population transfer in the desired state. Plot a 2 shows the converged optimized electric field as a function of time. The maximum amplitude of the electric field is $0.0051 E_{h} / e a_{0}$. The frequency distribution of the optimized field is shown in plot a3. The power spectrum shows the amplitude for the major component of the optimized electric field to be of frequency $1014 \mathrm{~cm}^{-1}$, while the amplitude for the component of frequency corresponding to $\mathrm{C}-\mathrm{O}$ stretch vibration (2005 $\mathrm{cm}^{-1}$ ) is considerably less, which implies a multiphoton nature of the excitation process. The mechanism of population transfer from initial vibrational state to final vibrational state with time is shown in plot a4. Small contributions from higher frequencies are also seen in plot a3. As time increases the population of $v=0$ state decreases while that of target vibrational state keeps on increasing. Up to time $32,000 \hbar / E_{h}$ the system is oscillating between these vibrational states and at the end of the pulse, the entire population is transferred to the target $v=5$ vibrational state.

(b) Transition: $v=0 \rightarrow v=1$ : In this case, we have propagated the system for $64,000 \hbar / E_{h}$ of time. The pulse length is divided in 32768 time steps. The initial guess laser field is chosen as $\epsilon(t)=0.0001 \cdot \sin \left(\omega_{0,5} t\right) s(t)$ where $\omega_{0,5}$ is the same transition frequency as chosen for previous transition. The convergence of transition probability and cost functional with the number of iterative steps shows (Fig. 3(b1)). The figure shows that the desired goal is achieved, but clearly population has been transferred to some more highly excited states during the excitation process. Further work is in progress to better characterise the excitation process and to try to find better electric fields to achieve the desired goal. The optimized electric field is shown in plot b2 of Fig. 3. The frequency spectrum of the optimized field shown in plot b3 is quite complex, which shows the contributions from many frequencies. The plot b4 shows the evolution of the populations of the lowest 6 vibrational states as a function of time.

\section{Conclusion}

Our optimal control calculations of vibrational excitations in the active part of the biologically important molecule carboxy-myoglobin provides laser fields which lead to selective vibrational excitations within the molecule, subject to the simplifications inherent in the model of the system. We have discussed our results for two vibrational excitation processes. The shape of the optimized electric field and its corresponding frequency spectrum is quite complex for the second transition considered, namely, $v=0 \longrightarrow v=1$ corresponding essentially to excitation of the Fe-C bond. We are able to achieve our dynamical goals with nearly $100 \%$ population transfer at the end of the pulse. The evolution of vibrational states involved in both the transitions show that there is some missing population. This population must have been transferred temporarily to higher 
lying vibrational states. Another pertinent issue is a change in the polarity of the triatomic for Fe-C bond distances beyond the range shown in Fig. 2. It is possible that this introduces some complexity into the molecule-radiation interaction. These aspect are being investigated further. Application of OCT to the photodissociation of the $\mathrm{Fe}-\mathrm{C}$ bond in carboxy-myoglobin is also in progress.

Acknowledgements. We thank the Royal Society and British Council, India, and DST, GOI, for supporting this work. SS thanks Council of Scientific and Industrial Research, Government of India for research fellowship.

\section{References}

1. Rice, S., Zhao, M.: Optical Control of Molecular Dynamics. John Wiley \& Sons, New York (2000)

2. Shapiro, M., Brumer, P.: Principles of the Quantum Control of Molecular Processes. John Wiley \& Sons, New York (2003)

3. Ohtsuki, Y., Nakagami, K., Fujimura, Y.: Quantum optimal control of multiple targets: Development of a monotonically convergent algorithm and application to intramolecular vibrational energy redistribution control. J. Chem. Phys. 114, 8867$8876(2001)$

4. Tannor, D.J., Rice, S.A.: Control of selectivity of chemical reaction via control of wave packet evolution. J. Chem. Phys. 83, 5013-5018 (1985)

5. Tannor, D.J., Kosloff, R., Rice, S.A.: Coherent pulse sequence induced control of selectivity of reactions: Exact quantum mechanical calculations. J. Chem. Phys. 85, 5805-5820 (1986)

6. Tannor, D.J., Rice, S.A.: Coherent pulse sequence control of product formation in chemical reactions. Adv. Chem. Phys. 70, 441-523 (1988)

7. Balint-Kurti, G.G., Manby, F.R., Ren, Q., Artamonov, M., Ho, T., Rabitz, H.: Quantum control of molecular motion including electronic polarization effects with a two-stage toolkit. J. Chem. Phys. 122, 84110 (2005); Ren, Q., Balint-Kurti, G.G., Manby, F.R., Artamonov, M., Ho, T., Rabitz, H.: Quantum control of molecular vibrational and rotational excitations in a homonuclear diatomic molecule: A full three-dimensional treatment with polarization forces. J. Chem. Phys. 124, 14111 (2006)

8. Shapiro, M., Brumer, P.: Laser Control of Molecular Processes. Ann. Rev. Phys. Chem. 43, 257-282 (1992)

9. Gross, P., Singh, H., Rabitz, H., Mease, K., Huang, G.M.: Inverse QuantumMechanical control: A mean for design and a test of intuition. Phys. Rev. A 47, 4593-4604 (1993)

10. Sharma, S., Sharma, P., Singh, H.: Quantum control of vibrational excitations in a heteronucler diatomic molecule. J. Chem. Sci. 119(2007), 433-440 (2007); Kumar, P., Singh, H.: Controlling dynamics in a diatomic systems. J. Chem. Sci. 119, 441-447 (2007)

11. Peirce, A.P., Dahleh, M.A., Rabitz, H.: Optimal control of quantum-mechanical systems: Existence, numerical approximation, and applications. Phys. Rev. A 37, 4950-4964 (1988)

12. Franzen, S.: An Electrostatic Model for the Frequency Shifts in the Carbonmonoxy Stretching Band of Myoglobin: Correlation of Hydrogen Bonding and the Stark Tuning Rate. J. Am. Chem. Soc. 124, 13271-13281 (2002) 
13. Dreuw, A., Duniez, B.D., Head-Gordon, M.: Characterization of the Relevant Excited States in the Photodissociation of CO-Ligated Hemoglobin and Myoglobin. J. Am. Chem. Soc. 124, 12070-12071 (2002)

14. Rovira, C., Schulze, B., Eichinger, M., Evanseck, J.D., Parrinello, M.: Influence of the Heme Pocket Conformation on the Structure and Vibrations of the Fe-CO Bond in Myoglobin: A QM/MM Density Functional Study. Biophys. J. 81, 435-445 (2001)

15. Meier, C., Heitz, M.C.: Laser control of vibrational excitation in carboxyhemoglobin: A quantum wave packet study. J. Chem. Phys. 123, 44504 (2005)

16. Ventalon, C., Fraser, J.M., Vos, M.H., Alexandrou, A., Martin, J.L., Joffre, M.: Coherent vibrational climbing in carboxyhemoglobin. Proc. Nat. Aac. Sci. 101, 13216-13220 (2004)

17. Frisch, M.J., et al.: Gaussian03, revison B.05, Gaussian, Inc., Pittsburgh P.A (2003)

18. Kachalova, G.S., Popov, A.N., Bartunik, H.D.: A Steric Mechanism for Inhibition of CO Binding to Heme. Prot. Sci. 284, 473-476 (1999)

19. Feit, M.D., Fleck Jr., J.A.: Solution of the Schrödinger equation by a spectral method II: Vibrational energy levels of triatomic molecules $a$ ). J. Chem. Phys. 78, 301-308 (1983)

20. Clay, C., Marston, Balint-Kurti, G.G.: The Fourier grid Hamiltonian method for bound state eigenvalues and eigenfunctions. J. Chem. Phys. 91, 3571-3578 (1989) 